\title{
Carbidopa and Levodopa Extended Release Capsules in Patients with and without Troublesome and Non-Troublesome Dyskinesia
}

\author{
Robert A. Hauser ${ }^{\mathrm{a}, *}$, Leonid Zeitlin ${ }^{\mathrm{b}}$, Stanley Fisher ${ }^{\mathrm{c}}$ and Richard D'Souza ${ }^{\mathrm{c}}$ \\ ${ }^{a}$ University of South Florida, Tampa, FL, USA \\ ${ }^{\mathrm{b}}$ Quartesian, Princeton, NJ, USA \\ ${ }^{\mathrm{c} A m n e a l}$ Pharmaceuticals, Bridgewater, NJ, USA
}

Accepted 27 May 2020

\begin{abstract}
.
Background: Carbidopa (CD) and levodopa (LD) extended release (CD-LD ER) capsules are designed to combine both immediate and extended release pharmacokinetics. In the phase 3, randomized, double-blind, ADVANCE-PD trial, patients randomized to CD-LD ER experienced a 1.17-hour greater reduction in OFF time compared to patients randomized to CD-LD $\operatorname{IR}(p<0.0001)$.

Objective: To compare CD-LD IR optimization to CD-LD ER conversion based on patient dyskinesia status at baseline using data from the ADVANCE-PD trial.

Methods: This was a retrospective analysis of the ADVANCE-PD study. Patients were categorized by dyskinesia status at baseline into 1) those who had No Dyskinesia (ND), 2) those who had Non-Troublesome Dyskinesia Only (NTDO), and 3) those who had Troublesome Dyskinesia (TD).

Results: Comparative reductions in OFF time favoring CD-LD ER over CD-LD IR were similar for the ND $(-1.08 \mathrm{~h}$, $p=0.0071, n=183)$ and NTDO $(-1.12 \mathrm{~h}, p=0.0104, n=131)$ groups, and smaller for the TD group $(-0.82 \mathrm{~h}, p=0.2382$, $n=79)$. Reductions in OFF time for both CD-LD ER conversion and CD-LD IR adjustment were largest within the ND group and smallest within the TD group (CD-LD ER: ND $-2.86 \mathrm{~h}$, NTDO $-2.11 \mathrm{~h}, \mathrm{TD}-1.36 \mathrm{~h}$; CD-LD IR: ND $-1.78 \mathrm{~h}$, NTDO $-0.99 \mathrm{~h}, \mathrm{TD}-0.55 \mathrm{~h})$.

Conclusion: Responses to both CD-LD IR adjustment and CD-LD ER conversion depended on baseline dyskinesia status. Significant reductions in OFF time with CD-LD ER compared to CD-LD IR were observed in the ND and NTDO groups. In the TD group, comparing CD-LD ER conversion to CD-LD IR optimization, benefits were still observed, but there was less reduction in OFF time, less reduction in troublesome dyskinesia, and fewer patients self-rated themselves much or very much improved than in the ND and NTDO groups. These data suggest that in clinical practice, the best chances for success with conversion from CD-LD IR to CD-LD ER are in patients without TD.
\end{abstract}

Keywords: Levodopa, extended release, Parkinson's disease, treatment, dyskinesia, OFF

\footnotetext{
*Correspondence to: Robert A. Hauser, MD, MBA, Parkinson's Disease and Movement Disorders Center, Parkinson Foundation Center of Excellence, University of South Florida, 4001 E. Fletcher
} 


\section{INTRODUCTION}

Carbidopa (CD) and levodopa (LD) extended release (CD-LD ER, Rytary ${ }^{\circledR}$ ) capsules are designed to combine both immediate and extended release pharmacokinetics. Upon ingestion of CD-LD ER, the initial increase in LD concentration is comparable to that of CD-LD IR [1]. After an initial peak at about one hour, plasma concentrations are maintained for about 4 to 5 hours before declining [1]. These pharmacokinetic properties allow for less frequent dosing and more sustained clinical benefit compared to other oral levodopa formulations.

The phase 3, randomized, double-blind trial, ADVANCE-PD, evaluated CD-LD ER vs CD-LD IR in Parkinson's disease (PD) patients with motor fluctuations [2]. Four hundred seventy-one PD patients with at least 2.5 hours of OFF time per day underwent 3 weeks of open label CD-LD IR dose adjustment followed by 6 weeks open label CD-LD ER dose conversion and adjustment (Fig. 1). Three hundred ninety-three patients who successfully completed these periods were then randomized to 13 weeks double-blind treatment with CD-LD IR or CD-LD ER plus placebo for the other using a double-dummy design. Results demonstrated that patients randomized to CD-LD ER experienced a 1.17-hour greater reduction in OFF time from baseline compared to patients randomized to CD-LD IR $(p<0.0001)$. In addition, patients received CD-LD ER an average of 3.6 times per day compared to 5.0 times per day for CD-LD IR. Troublesome dyskinesia was not significantly increased with CD-LD ER vs CD-LD IR. Patients randomized to CD-LD ER also exhibited significantly better patient global impression of change (PGI-C), clinician global impression of change (CGI-C), Unified Parkinson's Disease Rating Scale (UPDRS) I, II, III, I+ II + III, and total 39-item Parkinson's Disease Questionnaire (PDQ39) scores. During the open label conversion from CD-LD IR to CD-LD ER, 5\% of patients withdrew due to adverse events and $3 \%$ withdrew due to lack of efficacy. The most common adverse events during conversion were dyskinesia $(6 \%)$, nausea $(5 \%)$, headache $(4 \%)$, and dizziness $(4 \%)$. At randomization, the mean daily levodopa dose of CD-LD ER was approximately twice that of the CD-LD IR group (1630 mg versus $814.5 \mathrm{mg}$ ).

In clinical trials of PD patients with motor fluctuations, patients are typically required to have a predefined minimum amount of OFF time, but the amount and degree of dyskinesia can vary widely

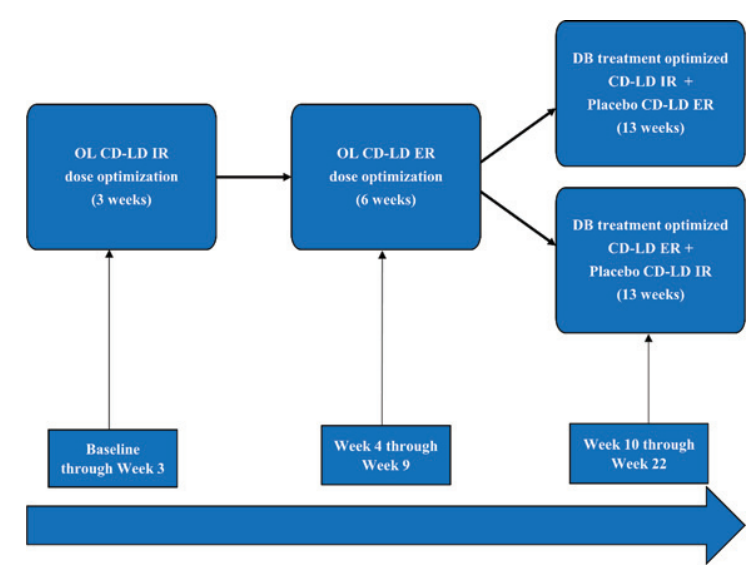

Fig. 1. Study Schematic. CD-LD ER, carbidopa-levodopa extended release; CD-LD IR, carbidopa-levodopa immediate release; DB, double-blind; OL, open label.

from patient to patient. In the current retrospective analysis of ADVANCE-PD, we sought to evaluate responses to CD-LD ER vs. CD-LD IR based on baseline dyskinesia status. Specifically, we sought to determine whether or not there was similar benefit observed for patients without dyskinesia, those with non-troublesome dyskinesia only, and those with troublesome dyskinesia.

\section{MATERIALS AND METHODS}

This was a retrospective analysis of the ADVANCE-PD study. The basic design of this study is described above, and full details of the study are available elsewhere [2]. All patients enrolled in the study had to have PD according to United Kingdom Parkinson's Disease Brain Bank criteria, were age 30 years or older at the time of diagnosis, Hoehn and Yahr stage I-IV in the ON state, treated with a stable regimen of CD-LD IR for at least 4 weeks before screening, and receiving a total daily LD dose of at least $400 \mathrm{mg}$ with a daily levodopa dosing frequency of at least 4 times per day, and experiencing an average of at least 2.5 hours OFF time per day as assessed on 3-day home PD diaries at baseline. The study excluded patients with severe dyskinesia at baseline (score $\geq 3$ [severely disabling] on item 33 of the UPDRS or a combined total $\geq 5$ on items 32,33 , and 34 ). The current analysis evaluated the 393 patients who were randomized into the 13-week double-blind treatment phase of the study. Patients were categorized by dyskinesia 
Table 1

Demographic and Baseline Disease Characteristics

\begin{tabular}{|c|c|c|c|}
\hline & $\begin{array}{l}\text { No Dyskinesia } \\
\qquad(\mathrm{N}=183)\end{array}$ & $\begin{array}{l}\text { Non-troublesome } \\
\text { Dyskinesia Only } \\
\quad(\mathrm{N}=131)\end{array}$ & $\begin{array}{c}\text { Troublesome } \\
\text { Dyskinesia } \\
(\mathrm{N}=79)\end{array}$ \\
\hline Age (years) & $64.1(9.81)$ & $63.7(9.05)$ & $60.5(8.65)$ \\
\hline Sex, male & $119(65.0)$ & $90(68.7)$ & $45(57.0)$ \\
\hline Parkinson's disease duration (years) & $6.06(4.023)$ & $8.24(4.641)$ & $9.23(4.311)$ \\
\hline UPDRS I + II + III & $34.6(15.93)$ & $33.8(14.54)$ & $33.9(15.40)$ \\
\hline Daily OFF time (hours) & $6.40(2.328)$ & $5.70(1.925)$ & $5.43(1.707)$ \\
\hline Daily ON-time with non-troublesome dyskinesia (hours) & $0.00(0.00)$ & $2.81(2.773)$ & $3.18(1.953)$ \\
\hline Daily ON-time with troublesome dyskinesia (hours) & $0.00(0.00)$ & $0.00(0.00)$ & $1.80(1.432)$ \\
\hline Daily ON-time without troublesome dyskinesia (hours) & $9.83(2.251)$ & $10.82(2.058)$ & $9.19(2.691)$ \\
\hline Daily levodopa dose (mg) & $756.4(376.70)$ & $770.1(333.80)$ & $830.1(326.66)$ \\
\hline Daily number of levodopa intakes & $4.7(1.13)$ & $5.1(1.39)$ & $5.7(2.31)$ \\
\hline
\end{tabular}

Data are Mean (SD) or n (\%). UPDRS, Unified Parkinson's Disease Rating Scale. UPDRS II and III are in the ON-state.

status at baseline, based on PD diaries [3] into 1) those who had no dyskinesia (No Dyskinesia [ND] group), 2) those who had at least one half-hour of non-troublesome dyskinesia, but no troublesome dyskinesia (Non-troublesome Dyskinesia Only [NTDO] group), and 3) those who had at least one half-hour of troublesome dyskinesia (Troublesome dyskinesia [TD] group).

Demographics and baseline characteristics were summarized descriptively with means and standard deviations (SD). Changes in daily dose and frequency of LD were summarized descriptively with means. Efficacy parameters were analyzed using statistical methodology consistent with the main analysis of the ADVANCE-PD study. For continuous parameters (such as diary measures, UPDRS and PDQ-39 scores) an ANCOVA model with change from baseline at end of study (EOS) as outcome, treatment and pooled site as fixed effects and baseline value as covariate, was used separately for each dyskinesia group. For PGI-C and CGI-C scores (that do not have baselines and are themselves measures of change) an ANOVA model with the score at EOS as outcome, treatment and pooled site as fixed effects was used. In both cases, the ANCOVA/ANOVA model was used to estimate least square (LS) means of the changes from baseline to EOS for each treatment group, as well as the difference in LS means, its confidence interval and the $p$-value for the test of no difference. For binary (yes/no) outcomes, counts and percentages of subjects were presented with a confidence interval for the difference in percentages between the treatment groups; Cochran-Mantel-Haenszel (CMH) test was performed to compare the proportions between the treatment groups.

\section{RESULTS}

\section{Demographics and baseline characteristics}

Of the 393 patients who entered the double-blind phase of the study, 183 had No Dyskinesia at baseline, 131 had Non-Troublesome Dyskinesia Only, and 79 had Troublesome Dyskinesia. Demographics and baseline characteristics of the three groups are displayed in Table 1. Concomitant PD medications are noted in Supplementary Table 1. Age at randomization was youngest and PD duration was longest for the TD group. In addition, both OFF time and $\mathrm{ON}$ time without troublesome dyskinesia were least for the TD group. At the same time, the TD group was receiving the highest daily LD dosage and had the highest number of daily LD administrations. Baseline UPDRS scores were similar across groups.

\section{Changes in levodopa dosages}

Changes in levodopa dosing regimens for the 3 groups are displayed in Table 2. Patients randomized to CD-LD IR received similar, slight increases in LD daily dosages across the 3 dyskinesia groups (1.05-1.07 times baseline daily levodopa dosage) and similar, very slight increases in the mean number of levodopa administrations per day $(0.1-0.2)$. However, whereas patients randomized to CD-LD ER received 2.25 and 2.24 times their baseline daily levodopa dosage in the ND and NTDO groups, respectively, patients in the TD group received a smaller increase at 2.07 times their baseline daily levodopa dosage. Notably, all 3 CD-LD ER groups 
Table 2

Levodopa Doses by Baseline Dyskinesia Status (Randomized Population)

\begin{tabular}{|c|c|c|c|c|c|c|}
\hline \multirow[b]{2}{*}{ Characteristic } & \multicolumn{2}{|c|}{ No Dyskinesia } & \multicolumn{2}{|c|}{$\begin{array}{l}\text { Non-Troublesome } \\
\text { Dyskinesia Only }\end{array}$} & \multicolumn{2}{|c|}{$\begin{array}{l}\text { Troublesome } \\
\text { Dyskinesia }\end{array}$} \\
\hline & $\begin{array}{l}\text { CD-LD ER } \\
(\mathrm{N}=91)\end{array}$ & $\begin{array}{c}\text { CD-LD IR } \\
(\mathrm{N}=92)\end{array}$ & $\begin{array}{l}\text { CD-LD ER } \\
(\mathrm{N}=66)\end{array}$ & $\begin{array}{l}\text { CD-LD IR } \\
(\mathrm{N}=65)\end{array}$ & $\begin{array}{l}\text { CD-LD ER } \\
(\mathrm{N}=44)\end{array}$ & $\begin{array}{c}\text { CD-LD IR } \\
(\mathrm{N}=35)\end{array}$ \\
\hline Mean LD Dose at Baseline (mg)* & 761.5 & 751.4 & 779.0 & 761.2 & 802.3 & 865.0 \\
\hline Mean LD Dose at EOS (mg) & 1611.6 & 788.9 & 1713.9 & 790.0 & 1541.8 & 927.1 \\
\hline $\begin{array}{l}\text { Mean Change from Baseline to EOS in } \\
\text { LD Dose }(\mathrm{mg})\end{array}$ & 850.1 & 37.5 & 935.0 & 28.8 & 739.5 & 62.1 \\
\hline $\begin{array}{l}\text { Ratio EOS LD Dose to Baseline LD } \\
\text { Dose }\end{array}$ & 2.25 & 1.07 & 2.24 & 1.05 & 2.07 & 1.06 \\
\hline $\begin{array}{l}\text { Mean Number of LD administrations at } \\
\text { Baseline }\end{array}$ & 4.8 & 4.6 & 5.1 & 5.1 & 6.0 & 5.4 \\
\hline $\begin{array}{l}\text { Mean Number of LD administrations at } \\
\text { EOS }\end{array}$ & 3.5 & 4.7 & 3.7 & 5.2 & 3.7 & 5.5 \\
\hline $\begin{array}{l}\text { Mean Change from Baseline to EOS in } \\
\text { Number of LD administrations }\end{array}$ & -1.3 & 0.1 & -1.3 & 0.2 & -2.3 & 0.1 \\
\hline $\begin{array}{l}\text { Ratio EOS Number of LD } \\
\text { administrations to Baseline Number of } \\
\text { LD administrations }\end{array}$ & 0.75 & 1.03 & 0.77 & 1.04 & 0.69 & 1.02 \\
\hline
\end{tabular}

experienced reductions in mean number of LD administrations per day, but this decrease was largest for patients in the TD group (ND -1.3, NTDO -1.3, TD -2.3) potentially related to their higher number of CD-LD IR administrations at baseline.

\section{Diary measures}

Diary changes from baseline to EOS by dyskinesia group, comparing CD-LD ER to CD-LD IR are presented in Table 3 and displayed in Fig. 2A and $\mathrm{B}$. There were significant reductions in OFF time with CD-LD ER compared to CD-LD IR in the ND $(-1.08 \mathrm{~h}, p=0.0071)$ and NTDO $(-1.12 \mathrm{~h}, p=0.0104)$ groups. The reduction in OFF time with CD-LD ER compared to CD-LD IR in the TD group was somewhat smaller than in the other dyskinesia groups, and not significant ( $-0.82 \mathrm{~h}, p=0.2382)$. Notably, reductions in OFF time for both CD-LD ER and CD-LD IR within each group were largest for the ND group and smallest for the TD group (CD-LD ER: ND $-2.86 \mathrm{~h}$, NTDO -2.11 h, TD $-1.36 \mathrm{~h}$; CD-LD IR: ND $-1.78 \mathrm{~h}$, NTDO $-0.99 \mathrm{~h}, \mathrm{TD}-0.55 \mathrm{~h}$ ).

Similarly, ON time without troublesome dyskinesia was significantly increased with CD-LD ER compared to CD-LD IR in the ND $(+0.90 \mathrm{~h}$, $p=0.0286)$ and NTDO $(+1.24 \mathrm{~h}, p=0.0105)$ groups but not the TD group $(-0.45 \mathrm{~h}, p=0.5609)$. Interestingly, in the TD group, troublesome dyskinesia was slightly reduced with CD-LD ER $(-0.16 \mathrm{~h})$ but more reduced with CD-LD IR $(-1.13 \mathrm{~h})$, although the difference between these was not statistically significant.

\section{PGI-C and CGI-C}

PGI-C and CGI-C data are presented in Table 3 and PGI-C score changes are depicted in Fig. 2C. Results indicated statistically significant benefit favoring CDLD ER over CD-LD IR for PGI-C, CGI-C, percent of patients self-rated as much or very much improved and percent of patients clinician-rated as much or very much improved across all three dyskinesia groups except PGI-C for the TD group where a favorable trend $(p=0.1174)$ was noted. Notably, even in the TD group, a significantly greater proportion of patients self-rated themselves much or very much improved with CD-LD ER (13/44, 29.5\%) compared to CD-LD $\operatorname{IR}(1 / 35,2.9 \% ; p=0.0026)$. For both CD-LD ER and CD-LD IR, the highest percentage of patients selfrated themselves as much or very much improved in the ND group and the lowest in the TD group (CD-LD ER: ND $46.7 \%$, NTDO $33.3 \%$, TD $29.5 \%$; CD-LD IR ND $25.6 \%$, NTDO $13.8 \%$, TD 2.9\%).

\section{$P D Q-39$}

Changes in PDQ-39 scores are displayed in Table 4 . There were statistically significant differences favoring CD-LD ER over CD-LD IR in the ND 
Table 3

Efficacy Outcomes (Patient Diary, PGI-C and CGI-C) by Baseline Dyskinesia Status (Change from Baseline to EOS) (Randomized Population)

\begin{tabular}{|c|c|c|c|c|c|c|c|c|c|c|c|c|}
\hline \multirow[b]{2}{*}{ Endpoint } & \multicolumn{4}{|c|}{ No Dyskinesia } & \multicolumn{4}{|c|}{ Non-Troublesome Dyskinesia Only } & \multicolumn{4}{|c|}{ Troublesome Dyskinesia } \\
\hline & $\begin{array}{c}\text { CD-LD } \\
\text { ER } \\
(\mathrm{N}=91)\end{array}$ & $\begin{array}{c}\text { CD-LD } \\
\text { IR } \\
(\mathrm{N}=92)\end{array}$ & $\begin{array}{l}\text { LS Mean } \\
\text { Difference }\end{array}$ & $\begin{array}{c}p- \\
\text { value }\end{array}$ & $\begin{array}{c}\text { CD-LD } \\
\text { ER } \\
(\mathrm{N}=66)\end{array}$ & $\begin{array}{c}\text { CD-LD } \\
\text { IR } \\
(\mathrm{N}=65)\end{array}$ & $\begin{array}{l}\text { LS Mean } \\
\text { Difference }\end{array}$ & $\begin{array}{c}p- \\
\text { value }\end{array}$ & $\begin{array}{c}\text { CD-LD } \\
\text { ER } \\
(\mathrm{N}=44)\end{array}$ & $\begin{array}{c}\text { CD-LD } \\
\text { IR } \\
(\mathrm{N}=35)\end{array}$ & $\begin{array}{l}\text { LS Mean } \\
\text { Difference }\end{array}$ & $\begin{array}{c}p- \\
\text { value }\end{array}$ \\
\hline \multicolumn{13}{|l|}{ Patient Diary } \\
\hline OFF-time, $\mathrm{h}$ & -2.86 & -1.78 & -1.08 & 0.0071 & -2.11 & -0.99 & -1.12 & 0.0104 & -1.36 & -0.55 & -0.82 & 0.2382 \\
\hline $\begin{array}{l}\text { ON-time without troublesome } \\
\text { dyskinesia, h }\end{array}$ & 2.66 & 1.76 & 0.90 & 0.0286 & 1.50 & 0.25 & 1.24 & 0.0105 & 1.40 & 1.85 & -0.45 & 0.5609 \\
\hline ON-time without dyskinesia, $\mathrm{h}$ & 2.35 & 1.35 & 1.01 & 0.0366 & 1.46 & 0.39 & 1.07 & 0.1272 & 1.16 & 2.16 & -1.00 & 0.2666 \\
\hline $\begin{array}{l}\text { ON-time with non-troublesome } \\
\text { dyskinesia, h }\end{array}$ & 0.31 & 0.41 & -0.10 & 0.6812 & 0.03 & -0.13 & 0.16 & 0.8063 & 0.08 & -0.17 & 0.25 & 0.7165 \\
\hline $\begin{array}{l}\text { ON-time with troublesome } \\
\text { dyskinesia, h }\end{array}$ & 0.20 & 0.05 & 0.15 & 0.3362 & 0.31 & 0.50 & -0.19 & 0.4693 & -0.16 & -1.13 & 0.98 & 0.1298 \\
\hline \multicolumn{13}{|l|}{$P G I-C$ and $C G I-C$} \\
\hline PGI-C (all participants) [2] & 5.25 & 4.59 & 0.66 & 0.0015 & 4.90 & 4.21 & 0.68 & 0.0112 & 4.46 & 3.84 & 0.62 & 0.1174 \\
\hline $\begin{array}{l}\text { Participants much or very much } \\
\text { improved [1] }\end{array}$ & $42(46.7 \%)$ & $23(25.6 \%)$ & 21.1 & 0.0033 & $22(33.3 \%)$ & $9(13.8 \%)$ & 19.5 & 0.0090 & $13(29.5 \%)$ & $1(2.9 \%)$ & 26.6 & 0.0026 \\
\hline CGI-C (all participants) [2] & 5.22 & 4.77 & 0.45 & 0.0085 & 4.83 & 4.27 & 0.56 & 0.0140 & 4.76 & 3.98 & 0.78 & 0.0300 \\
\hline $\begin{array}{l}\text { Participants much or very much } \\
\text { improved [1] }\end{array}$ & $41(45.6 \%)$ & $17(18.9 \%)$ & 26.7 & 0.0001 & $22(33.3 \%)$ & $7(10.8 \%)$ & 22.6 & 0.0019 & $17(38.6 \%)$ & $2(5.7 \%)$ & 32.9 & 0.0007 \\
\hline
\end{tabular}

Note: LS Means and $p$-value from an ANCOVA model with change from baseline at EOS as outcome, treatment and pooled site as fixed effects and baseline value as covariate, unless otherwise specified. [1] Counts and percentages of subjects and $p$-value from the CMH test. [2] LS Means and $p$-value from an ANOVA model with PGI-C or CGI-C score at EOS as outcome, treatment and pooled site as fixed effects. ANCOVA, Analysis of Covariance; ANOVA, Analysis of Variance; CD-LD ER, carbidopa-levodopa extended release; CD-LD IR, carbidopa-levodopa immediate release; CGI-C, Clinical Global Impression of Change; CMH, Cochran-Mantel-Haenszel; EOS, End of Study; h, hour; LS, least squares; PDQ-39, 39-Item Parkinson's Disease Questionnaire; PGI-C, Patient Global Impression of Change; UPDRS, Unified Parkinson's Disease Rating Scale. 

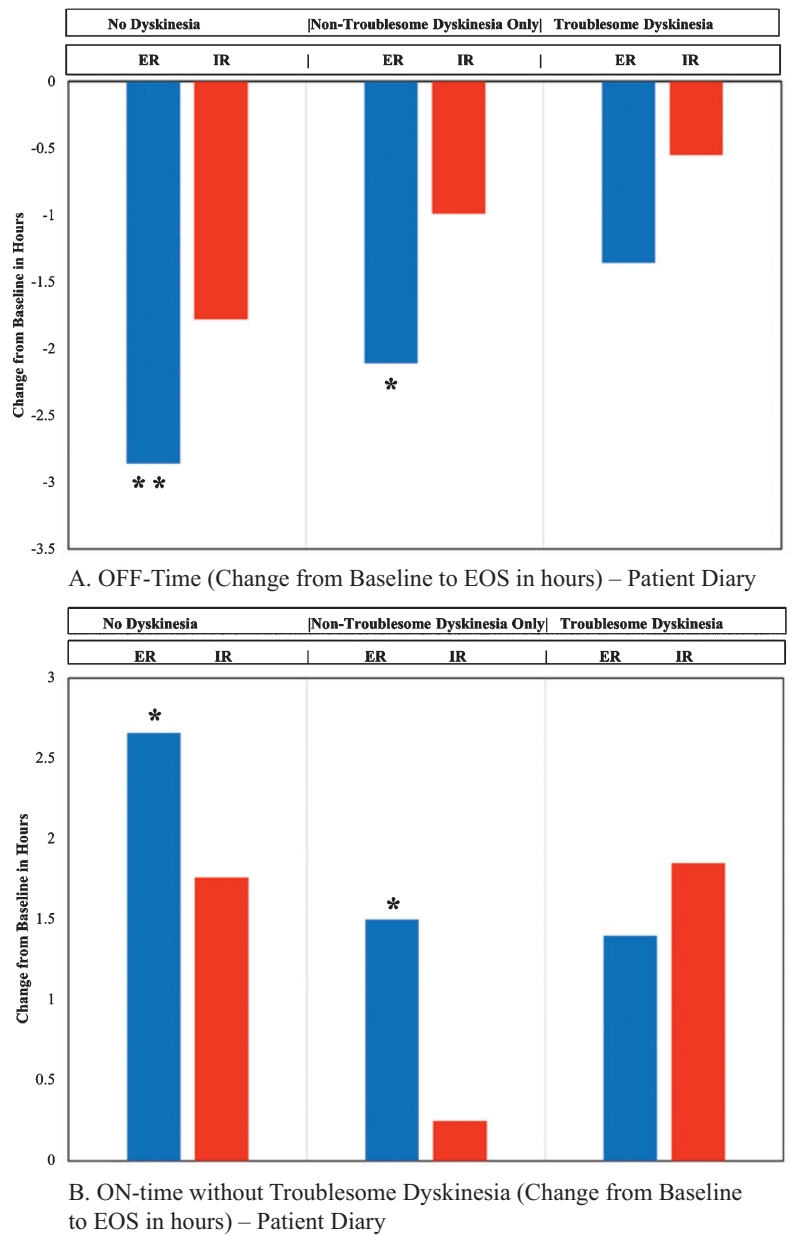

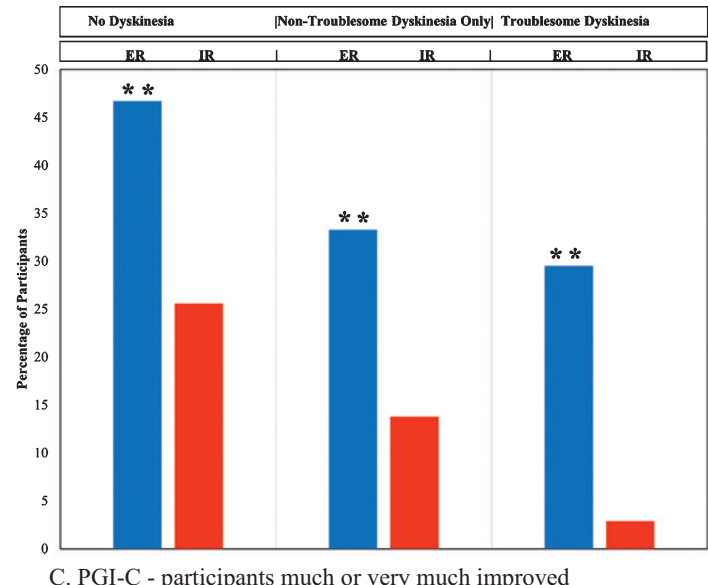

C. PGI-C - participants much or very much improved

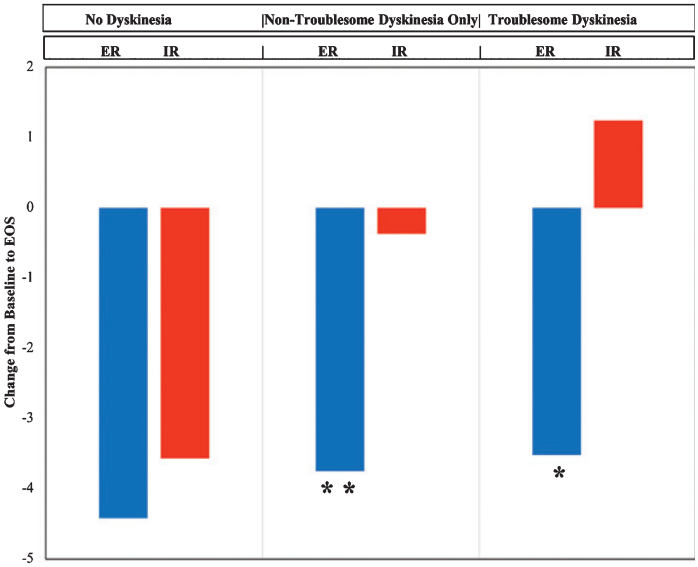

D. UPDRS Part III

Fig. 2. Selected Efficacy Outcome Measures. *denotes $p$-value $<0.05$ and statistically significant difference in the ER group as compared to the IR group. **denotes $p$-value $<0.01$ and statistically significant difference in the ER group as compared to the IR group. EOS, End of Study; ER, extended release; IR, immediate release; PGI-C, Patient Global Impression of Change; UPDRS, Unified Parkinson's Disease Rating Scale.

group for total score, mobility and activities of daily living.

\section{UPDRS scores}

UPDRS score changes are presented in Table 4 and displayed in Fig. 2D. CD-LD ER was associated with significantly more improved UPDRS II ON scores compared to CD-LD IR in the ND group. UPDRS III scores were essentially similarly improved with CD-LD ER across all three dyskinesia groups (ND -4.42, NTDO -3.75, TD -3.52). However, UPDRS III scores were most improved with CD-LD IR in the ND group and the least improved (worsened) in the TD group (ND -3.57, NTDO -0.37, TD + 1.24). Relative differences across groups lead to statistically significant benefits in favor of CD-LD ER over CD-LD IR for change in UPDRS III scores in the
NTDO and TD groups but not the ND group (ND $-0.85 p=0.4160, \mathrm{NTDO}-3.38 p=0.0047, \mathrm{TD}-4.76$ $p=0.0299$ ).

\section{Safety}

The incidences of the most common adverse events (AEs) during the 13-week double-blind maintenance period are displayed in Table 5. Overall, subjects randomized to CD-LD ER vs CD-LD IR experienced similar rates of AEs in the ND (ER $40.7 \%$ vs IR $38.0 \%$ ) and NTDO (ER $42.4 \%$ vs IR $43.1 \%$ ) groups but in the TD group, AEs were more frequent in subjects randomized to CD-LD ER (CD-LD ER 50.0\% vs CD-LD IR 37.1\%). In the TD group, falls were reported in 4 of 44 patients $(9.1 \%)$ randomized to CD-LD ER and in 1 of 35 (2.9\%) randomized to $\mathrm{CD}-\mathrm{LD}$ IR. 
Table 4

Efficacy Outcomes (UPDRS and PDQ-39) by Baseline Dyskinesia Status (Change from Baseline to EOS) (Randomized Population)

\begin{tabular}{|c|c|c|c|c|c|c|c|c|c|c|c|c|}
\hline \multirow[b]{2}{*}{ Endpoint } & \multicolumn{4}{|c|}{ No Dyskinesia } & \multicolumn{4}{|c|}{ Non-Troublesome Dyskinesia Only } & \multicolumn{4}{|c|}{ Troublesome Dyskinesia } \\
\hline & $\begin{array}{c}\text { CD-LD } \\
\text { ER } \\
(\mathrm{N}=91)\end{array}$ & $\begin{array}{c}\text { CD-LD } \\
\text { IR } \\
(\mathrm{N}=92)\end{array}$ & $\begin{array}{c}\text { LS Mean } \\
\text { Difference }\end{array}$ & $\begin{array}{c}p- \\
\text { value }\end{array}$ & $\begin{array}{c}\text { CD-LD } \\
\text { ER } \\
(\mathrm{N}=66)\end{array}$ & $\begin{array}{c}\text { CD-LD } \\
\text { IR } \\
(\mathrm{N}=65)\end{array}$ & $\begin{array}{c}\text { LS Mean } \\
\text { Difference }\end{array}$ & $\begin{array}{c}p- \\
\text { value }\end{array}$ & $\begin{array}{c}\text { CD-LD } \\
\text { ER } \\
(\mathrm{N}=44)\end{array}$ & $\begin{array}{c}\text { CD-LD } \\
\text { IR } \\
(\mathrm{N}=35)\end{array}$ & $\begin{array}{c}\text { LS Mean } \\
\text { Difference }\end{array}$ & $\begin{array}{c}p- \\
\text { value }\end{array}$ \\
\hline \multicolumn{13}{|l|}{$\overline{U P D R S}$} \\
\hline Parts II and III & -6.15 & -4.23 & -1.92 & 0.1587 & -4.54 & -1.24 & -3.31 & 0.0576 & -4.56 & 2.29 & -6.85 & 0.0191 \\
\hline Parts I, II, and III & -6.40 & -4.14 & -2.27 & 0.1157 & -4.84 & -1.29 & -3.55 & 0.0495 & -4.83 & 2.42 & -7.25 & 0.0195 \\
\hline Total & -6.69 & -4.64 & -2.05 & 0.1811 & -5.69 & -1.37 & -4.32 & 0.0262 & -4.90 & 2.03 & -6.94 & 0.0320 \\
\hline Part I & -0.25 & 0.10 & -0.35 & 0.0636 & -0.29 & -0.04 & -0.25 & 0.3031 & -0.37 & 0.11 & -0.48 & 0.2793 \\
\hline Part II: on state & -1.64 & -0.55 & -1.10 & 0.0423 & -0.69 & -0.73 & 0.04 & 0.9625 & -1.00 & 0.92 & -1.92 & 0.1068 \\
\hline Part II: off state & -1.70 & -0.64 & -1.06 & 0.0803 & -0.50 & -0.52 & 0.01 & 0.9880 & -0.33 & 0.57 & -0.91 & 0.4547 \\
\hline Part III & -4.42 & -3.57 & -0.85 & 0.4160 & -3.75 & -0.37 & -3.38 & 0.0047 & -3.52 & 1.24 & -4.76 & 0.0299 \\
\hline Part IV & -0.27 & -0.36 & 0.09 & 0.7729 & -0.84 & -0.28 & -0.56 & 0.1718 & -0.12 & -0.38 & 0.26 & 0.6168 \\
\hline \multicolumn{13}{|l|}{$P D Q-39$} \\
\hline Total & -5.76 & -2.00 & -3.76 & 0.0192 & -2.33 & -1.74 & -0.59 & 0.8063 & -2.89 & -2.31 & -0.58 & 0.8428 \\
\hline Mobility & -7.01 & -1.45 & -5.56 & 0.0058 & -4.10 & -1.53 & -2.56 & 0.4532 & -2.67 & -2.64 & -0.04 & 0.9937 \\
\hline Activities of daily living & -9.47 & -3.42 & -6.05 & 0.0038 & -2.07 & -3.77 & 1.69 & 0.6165 & -0.34 & -2.38 & 2.04 & 0.6593 \\
\hline Emotional wellbeing & -3.30 & 0.22 & -3.53 & 0.1420 & -3.40 & -1.77 & -1.62 & 0.5268 & -2.90 & -6.49 & 3.60 & 0.2868 \\
\hline Stigma & -8.24 & -7.36 & -0.88 & 0.7000 & -3.19 & -4.31 & 1.12 & 0.7185 & -5.48 & -4.26 & -1.22 & 0.7700 \\
\hline Social support & -1.17 & 0.65 & -1.82 & 0.4399 & 1.00 & 0.84 & 0.16 & 0.9565 & -5.73 & -3.38 & -2.35 & 0.5165 \\
\hline Cognition & -0.93 & 0.64 & -1.58 & 0.4119 & -1.03 & -0.72 & -0.31 & 0.8933 & -4.43 & 1.61 & -6.04 & 0.1641 \\
\hline Communication & -3.39 & -1.03 & -2.36 & 0.3156 & -3.83 & -0.68 & -3.15 & 0.3084 & -3.12 & 2.39 & -5.51 & 0.2481 \\
\hline Bodily discomfort & -6.27 & -2.96 & -3.31 & 0.1760 & -2.12 & -2.75 & 0.63 & 0.8601 & -7.12 & 3.38 & -10.50 & 0.0906 \\
\hline
\end{tabular}

Note: LS Means and $p$-value from an ANCOVA model with change from baseline at EOS as outcome, treatment and pooled site as fixed effects and baseline value as covariate, unless otherwise specified. CD-LD ER, carbidopa-levodopa extended release; CD-LD IR, carbidopa-levodopa immediate release; EOS, End of Study; LS, least squares; PDQ-39, 39-Item Parkinson's Disease Questionnaire; UPDRS, Unified Parkinson's Disease Rating Scale. 
Table 5

Most Common Treatment-Emergent Adverse Events by Baseline Dyskinesia Status During the Double-Blind Maintenance Period (Randomized Population)

\begin{tabular}{|c|c|c|c|c|c|c|}
\hline \multirow[t]{2}{*}{ Preferred Term } & \multicolumn{2}{|c|}{ No Dyskinesia } & \multicolumn{2}{|c|}{ Non-Troublesome Dyskinesia Only } & \multicolumn{2}{|c|}{ Troublesome Dyskinesia } \\
\hline & $\begin{array}{c}\text { CD-LD ER } \\
(\mathrm{N}=91) \\
\mathrm{n}(\%)\end{array}$ & $\begin{array}{c}\text { CD-LD IR } \\
(\mathrm{N}=92) \\
\mathrm{n}(\%)\end{array}$ & $\begin{array}{c}\text { CD-LD ER } \\
(\mathrm{N}=66) \\
\mathrm{n}(\%)\end{array}$ & $\begin{array}{c}\text { CD-LD IR } \\
(\mathrm{N}=65) \\
\mathrm{n}(\%)\end{array}$ & $\begin{array}{c}\text { CD-LD ER } \\
(\mathrm{N}=44) \\
\mathrm{n}(\%)\end{array}$ & $\begin{array}{c}\text { CD-LD IR } \\
(\mathrm{N}=35) \\
\mathrm{n}(\%)\end{array}$ \\
\hline Subjects with at least one event & $37(40.7)$ & $35(38.0)$ & $28(42.4)$ & $28(43.1)$ & $22(50.0)$ & $13(37.1)$ \\
\hline Fall & $1(1.1)$ & $0(0.0)$ & $1(1.5)$ & $3(4.6)$ & $4(9.1)$ & $1(2.9)$ \\
\hline Insomnia & $2(2.2)$ & $1(1.1)$ & $4(6.1)$ & $1(1.5)$ & $1(2.3)$ & $0(0.0)$ \\
\hline Nausea & $5(5.5)$ & $2(2.2)$ & $1(1.5)$ & $0(0.0)$ & $0(0.0)$ & $1(2.9)$ \\
\hline Oedema peripheral & $2(2.2)$ & $1(1.1)$ & $2(3.0)$ & $3(4.6)$ & $0(0.0)$ & $0(0.0)$ \\
\hline Sleep disorder & $2(2.2)$ & $2(2.2)$ & $2(3.0)$ & $1(1.5)$ & $0(0.0)$ & $1(2.9)$ \\
\hline Upper respiratory tract infection & $2(2.2)$ & $3(3.3)$ & $0(0.0)$ & $1(1.5)$ & $2(4.5)$ & $0(0.0)$ \\
\hline Urinary tract infection & $1(1.1)$ & $3(3.3)$ & $1(1.5)$ & $1(1.5)$ & $2(4.5)$ & $0(0.0)$ \\
\hline Back pain & $1(1.1)$ & $1(1.1)$ & $1(1.5)$ & $3(4.6)$ & $1(2.3)$ & $0(0.0)$ \\
\hline Dizziness & $2(2.2)$ & $2(2.2)$ & $3(4.5)$ & $0(0.0)$ & $0(0.0)$ & $0(0.0)$ \\
\hline Dyskinesia & $1(1.1)$ & $0(0.0)$ & $2(3.0)$ & $1(1.5)$ & $2(4.5)$ & $1(2.9)$ \\
\hline
\end{tabular}

Note: Adverse events were coded according to the Medical Dictionary for Regulatory Activities version 12.1. If subject had multiple events with the same preferred term, the subject was counted only once. CD-LD ER, carbidopa-levodopa extended release; CD-LD IR, carbidopa-levodopa immediate release.

\section{DISCUSSION}

To our knowledge, this is the first report to analyze results of a Parkinson's disease clinical trial based on categorizing patients at baseline as having No Dyskinesia (ND), Non-troublesome Dyskinesia Only (NTDO), or Trouble some Dyskinesia (TD). We hypothesized that these groups may respond differently to various treatments both in clinical trials and in the clinical practice setting. In the current analysis we retrospectively analyzed the ADVANCE-PD trial that evaluated CD-LD IR optimization vs CD-LD ER conversion in patients with motor fluctuations, based on these dyskinesia categories.

Analysis of baseline demographics and characteristics showed that whereas the ND group was the oldest, had the shortest duration of PD, the lowest daily levodopa dose and the lowest number of daily levodopa administrations, they also had the greatest amount of daily OFF time. Conversely, the TD group was the youngest, had the longest duration of PD, the highest daily levodopa dose and the highest number of daily levodopa administrations, they also had the least amount of OFF time. Because the TD group also had troublesome dyskinesia (by definition), they also had the smallest amount of ON time without troublesome dyskinesia at baseline of the three dyskinesia category groups. These findings are consistent with those of others who noted that younger age and longer disease duration are risk factors for dyskinesia generally [4].

Patients with both OFF time and dyskinesia might be the most difficult to improve (by diary mea- sures) in classic PD motor fluctuator trials that assess the addition of adjunctive medications that increase dopaminergic stimulation. In these patients, an increase in dopaminergic stimulation may increase dyskinesia and either lead to an increase in troublesome dyskinesia or require a reduction in baseline levodopa dosage. Benefit might be more likely with treatments that either reduce levodopa concentration fluctuations or include an anti-dyskinesia mechanism. Since CD-LD ER smooths the LD concentration-time curve and reduces variability in LD concentrations compared with CD-LD IR [1], it may provide better results in patients with both OFF and dyskinesia than other products without similar characteristics.

Our analysis found that CD-LD ER provided significantly greater reductions in OFF time and increases in ON time without troublesome dyskinesia than CD-LD IR in both the ND and the NTDO groups. Although the differences observed between CD-LD ER and CD-LD IR were similar in the ND (OFF $-1.08 \mathrm{~h}$, ON time without troublesome dyskinesia [ONWoTD] $+0.9 \mathrm{~h}$ ) and NTDO (OFF -1.12 , ONWoTD $+1.24 \mathrm{~h}$ ) groups, the magnitude of changes for both CD-LD ER and CD-LD IR were greater within the ND group (OFF: ER $-2.86 \mathrm{~h}$, IR $-1.78 \mathrm{~h}$; ONWoTD: ER $+2.66 \mathrm{~h}, \mathrm{IR}+1.76 \mathrm{~h}$ ) than the NTDO group (OFF: ER $-2.11 \mathrm{~h}, \mathrm{IR}-0.99 \mathrm{~h}$; ONWoTD: $\mathrm{ER}+1.50 \mathrm{~h}, \mathrm{IR}+0.25 \mathrm{~h}$ ). Thus, on key diary measures, more benefit was obtained with CDLD IR adjustment alone in the ND group compared to the NTDO group, and more benefit was obtained with CD-LD ER conversion in the ND group compared to 
the NTDO group, but in each case, significantly more benefit was obtained with conversion to CD-LD ER than with CD-LD IR adjustment alone.

PGI-C and CGI-C scores followed a similar pattern and reflected significantly greater benefit with CDLD ER than CD-LD IR in both the ND and NTDO groups. Again, the magnitude of benefit observed was greater for both CD-LD ER and CD-LD IR in the ND group compared to the NTDO group.

Thus, clear benefits were observed with CD-LD ER compared to CD-LD IR in both the ND and NTDO groups. These benefits were achieved while reducing the number of daily levodopa administrations by 1.3 in the CD-LD ER groups compared to increases of 0.1 (ND) and 0.2 (NTDO) in the CD-LD IR groups.

One apparent difference in outcomes was seen in PDQ-39 total, mobility, and ADL scores, where significant benefit was observed for CD-LD ER compared to CD-LD IR in the ND group but not in the NTDO group. The reason for this difference is not clear. It could potentially relate to the greater magnitude of decrease in OFF time and increase in ON time without troublesome dyskinesia in the ND group. Another possibility is that these diary changes have a greater impact (on PDQ-39 scores) in patients with milder disease.

Results were mixed when comparing CD-LD ER and CD-LD IR in patients with TD. Among patients randomized to CD-LD ER, those in the TD group experienced the lowest dose conversion ratio from CD-LD IR at baseline to CD-LD ER at EOS (ND $2.25 \times$, NTDO $2.24 \times$, TD $2.07 \times$ ), and the greatest reduction in the number of daily levodopa administrations (ND -1.3, NTDO -1.3, TD -2.3). The TD group also yielded the smallest reduction in OFF time comparing CD-LD ER to CD-LD IR $(-0.82 \mathrm{~h}$, NS). Troublesome dyskinesia was also decreased by a smaller margin $(0.98 \mathrm{~h}$ less) with CD-LD ER compared to CD-LD IR in the TD group; troublesome dyskinesia was little changed with CD-LD ER $(-0.16 \mathrm{~h})$ and robustly decreased with CD-LD IR $(-1.13 \mathrm{~h})$. However, UPDRS III and II + III scores were significantly improved with CD-LD ER compared to CD-LD IR in the TD group as were CGI-C scores. Thus, overall, in the TD group, comparing CD-LD ER conversion to CD-LD IR optimization, benefit was still observed, but there was less reduction in OFF time, less reduction in troublesome dyskinesia, and fewer patients self-rated themselves much or very much improved.

Why UPDRS III scores were more improved with CD-LD ER compared to CD-LD IR in the TD group than in the ND group or NTDO group is not entirely clear. It appears this may relate to a worsening of UPDRS III scores with CD-LD IR in this group and this would go along with the observed reduction in troublesome dyskinesia in this group (although the increase in the CD-LD IR dose from baseline to EOS was slightly more in the TD group [+62.1 mg] than in the ND [+37.5 mg] and NTDO [+28.8 mg] groups).

Adverse events were somewhat more common with CD-LD ER than CD-LD IR (50.0\% vs $37.1 \%)$ in the TD group whereas they were similar in the ND and NTDO groups. This might reflect a more fragile population and one that is more prone to AEs related to dyskinesia. Indeed, falls were more common with CD-LD ER than CD-LD IR in the TD group and dyskinesia is a known risk factor for falls [5].

We note that our analysis has several limitations. This was a retrospective analysis and no power calculations were performed. The TD group had fewer patients than the ND and NTDO groups and this may have affected power for statistical analyses. In addition, the original trial excluded patients with severely disabling dyskinesia. Further, there were minor differences across groups in concomitant PD medications, and although these medications remained unchanged through the study, their effect on our results is unclear. We also note that the ND group experienced an increase in $\mathrm{ON}$ time with nontroublesome dyskinesia and a slight increase in $\mathrm{ON}$ time with troublesome dyskinesia with both CDLD ER and CD-LD IR dose optimization and the NTDO group experienced an increase in ON time with troublesome dyskinesia with both CD-LD ER and CD-LD IR dose optimization. This suggests that these groups were somewhat undertreated at baseline and that categorization of dyskinesia status for some patients depends on treatment status. Nonetheless, clinicians frequently need to make treatment decisions in patients who have not yet been optimized on their medication regimen.

Our analysis may have important implications for clinical practice as we demonstrated differences in response to both CD-LD IR adjustment and CD-LD ER conversion in patients with motor fluctuations depending on baseline dyskinesia status. The most benefit was achieved with CD-LD IR adjustment or CD-LD ER conversion in patients without dyskinesia and the least in patients with troublesome dyskinesia. However, in patients with No Dyskinesia or NonTroublesome Dyskinesia Only, greater benefit was observed with CD-LD ER than CD-LD IR. While we did observe benefit in the TD group with conversion 
to CD-LD ER compared to CD-LD IR adjustment in some outcome measures (including PGI-C), the overall benefit was less clear, and we suggest clinicians appropriately adjust expectations for success in this group. Our data suggest that in clinical practice, the best chances for success with conversion to CD-LD ER are in patients without troublesome dyskinesia.

\section{ACKNOWLEDGMENTS}

This study was funded by Amneal Pharmaceuticals.

\section{CONFLICT OF INTEREST}

RAH has received fees from Amneal Pharmaceuticals for consulting work and as a speaker. LZ is employed by Quartesian who was paid to perform the statistical analysis for this study. Quartesian prepared the tables and figures. SF and RD are employees of Amneal Pharmaceuticals.

In addition, Dr. Robert Hauser reports receiving consulting fees from: AbbVie, Academy for Continued Healthcare Learning, Acadia Pharmaceuticals, Acorda Therapeutics, Adamas Pharmaceuticals, Affriris, Alliance for Aging Research, Alphasights, Amneal Pharmaceuticals Inc., ApoPharma, Aptis Partners LLC, Aranca, Axial Biotherapeutics, Axovant Sciences, Bain Capital, Baron Capital, Brittanna Pharmaceuticals, Ltd., Cadent Therapeutics, Cerespir Inc., Clearview Healthcare Partners, CNS Ratings LLC., Compass Group, DOB Health LLC, Decision Resources Group (DRG), Defined Health, Dellaus Consulting, Denali Therapeutics, Enterin Inc, Evercore, Extera Partners, GE Healthcare, Gerson Lehrman Group (GLG), Global Kinetics Corporation, Guide Point Global, Health and Wellness Partners, Healthlogix, Heptares Therapeutics, Huron Consulting Group, Impax Laboratories, Impel Neuropharma, Inhibikase, Intec Pharma LTD, International Stem Cell Corporation, Intramed Educational Group, IQVIA, Jazz Pharmaceutics, Kaiser, Kyowa Kirin Pharmaceutical Development, Kashiv Pharma LLC, L.E.K. Consulting, Lundbeck, Lundbeck A/S, MedaCorp, MEDIQ, Medscape, Medtronic, Michael J Fox Foundation, Mitsubishi Tanabe Pharmaceuticals, Movement Disorder Society, Neuro Challenge Foundation for Parkinson's, Neurocea LLC, Neurocrine Biosciences, Neuroderm,
Northwestern University, Orbes Inc., Orbes Medical Group, Orion, Parkinson's Foundation, Parkinson Study Group, Partner's Healthcare, Penn Technology Partnership, Pennside Partners, Perception OpCo, Precision Effect, Phase Five Communications, Prescott Medical Group, Prilenia Therapeutics LLC, Projects in Knowledge, Regenera Pharma, SAi Med Partners LLC, Schlesinger Associates, Scion Neurostim LLC, Seagrove Partners, Seelos Therapeutics, Slingshot Insights, Sun Pharma, Sunovion Pharmaceuticals Inc, Teva Pharmaceuticals, The Lockwood Group, USWorldMeds, WebMD, and Windrose Consulting Group.

Dr. Hauser participated company sponsored speaker's bureaus: Acorda Therapeutics, Adamas Pharmaceuticals, Amneal Pharmaceuticals Inc., Kyowa Kirin Pharmaceutical Development, Neurocrine Biosciences and US WorldMeds.

Dr. Hauser reports research support from:AbbVie Inc, Acorda Therapeutics, AstraZeneca, Axovant Sciences, Biogen Inc, Cavion, Centogene, Cerevance, Cerevel, Covance, Enterin Inc, Global Kinetics Corp., Impax Laboratories LLC, Intec Pharma Ltd, Jazz Pharmaceuticals, NeuroDerm Ltd., Lundbeck, Michael J Fox Foundation for Parkinson's Research, Neuraly, Pharma2B, F. Hoffman-La Roche, Revance Therapeutics Inc, and Sunovion Pharmaceuticals.

Dr. Hauser reports grant support from: Parkinson Foundation.

Dr. Hauser has Stock Option Ownership in: Axial Biotherapeutics, Inhibikase Therapeutics Inc.

\section{SUPPLEMENTARY MATERIAL}

The supplementary material is available in the electronic version of this article: https://dx.doi.org/ 10.3233/JPD-202010.

\section{REFERENCES}

[1] Hsu A, Yao HM, Gupta S, Modi NB (2015) Comparison of the pharmacokinetics of an oral extendedrelease capsule formulation of carbidopa-levodopa (IPX066) with immediate-release carbidopa-levodopa (Sinemet $\left({ }^{\circledR}\right)$ ), sustained-release carbidopa-levodopa (Sinemet $\left.\left({ }^{\circledR}\right) \mathrm{CR}\right)$, and carbidopa-levodopa-entacapone (Stalevo $\left.\left({ }^{\circledR}\right)\right)$. J Clin Pharmacol 55, 995-1003.

[2] Hauser RA, Hsu A, Kell S, Espay AJ, Sethi K, Stacy M, Ondo W, O'Connell M, Gupta S; IPX066 ADVANCE-PD investigators (2013) Extended-release carbidopa-levodopa (IPX066) compared with immediate-release carbidopalevodopa in patients with Parkinson's disease and motor fluctuations: A phase 3 randomised, double-blind trial. Lancet Neurol 12, 346-356. 
[3] Hauser RA, Friedlander J, Zesiewicz TA, Adler CH, Seeberger LC, O'Brien CF, Molho ES, Factor SA (2000) A home diary to assess functional status in patients with Parkinson's disease with motor fluctuations and dyskinesia. Clin Neuropharmacol 23, 75-81.

[4] Zhou X, Guo J, Sun Q, Xu Q, Pan H, Yu R, Tan J, Yan X, Tang B, Fang L (2019) Factors associated with dyskinesia in Parkinson's disease in mainland China. Front Neurol 10, 477.
[5] Rascol O, Perez-Lloret S, Damier P, Delval A, Derkinderen P, Destée A, Meissner WG, Tison F, Negre-Pages L (2015) Falls in ambulatory non-demented patients with Parkinson's disease. J Neural Transm (Vienna) 122, 1447-1455. 